\title{
Ein einfaches Modell zum Vergleich der Theorien von Hauser-Feshbach und P. A. Moldauer
}

\author{
F. SCHMIDT * \\ Institut für Kernenergetik der Universität Stuttgart ** \\ (Z. Naturforsch. 23 a, 955-961 [1968] ; eingegangen am 5. Dezember 1967)
}

\begin{abstract}
We discuss and compare the theories of Hauser and Feshbach and P. A. Moldauer by a simple two channel model. It is found that after a good fit of parameters only the cross sections of compound-elastic scattering differ substantially. Most differences appear if there are a few open channels. The consequences for the cross section of compound nucleus formation and also for calculations by the evaporation mdel are considered. By our model an evident foundation is given for the statement that Moldauer's theorie would be valid for $\left\langle\Gamma_{\lambda c}\right\rangle \mid D \leqq 1$.
\end{abstract}

\section{Voraussetzungen}

Die beiden am häufigsten zur Berechnung von Wirkungsquerschnitten des über den Compoundkern verlaufenden Anteils der inelastischen Streuung schneller Neutronen verwendeten Theorien sind die von Hauser und Feshbach ${ }^{1}$ bzw. Moldauer ${ }^{2-4}$. Wir haben früher ${ }^{5,6}$ gezeigt, daß sie sich als verschiedene Näherungen derselben Theorie darstellen lassen, wobei die komplizierte Moldauersche Formulierung die vom höheren Grad ist.

Der Bohrschen Vorstellung entsprechend setzen die zu berechnenden Wirkungsquerschnitte sich aus dem Querschnitt $\sigma_{c}$ für die Bildung des Compoundkernes durch den Einschlußkanal $c$ und der Wahrscheinlichkeit für seinen Zerfall durch einen Kanal $c^{\prime}$ zusammen. Die Zerfallswahrscheinlichkeit kann dabei durch das Verhältnis der mittleren Breiten des Kanals $c^{\prime}\left\langle\Gamma_{2, c^{\prime}}\right\rangle$ und der totalen Breite

$$
\left\langle\Gamma_{\lambda}\right\rangle=\sum_{c^{\prime \prime}}\left\langle\Gamma_{\lambda c^{\prime \prime}}\right\rangle
$$

angegeben werden, so daß man für den Wirkungsquerschnitt, solange man nur annimmt, Ein- und Ausgangskanal seien nicht miteinander gekoppelt, erhält:

$$
\sigma_{c c^{\prime}}=\sigma_{c} \cdot\left\langle\Gamma_{\lambda c^{\prime}}\right\rangle /\left\langle\Gamma_{\lambda}\right\rangle
$$

Für $\sigma_{c}$ ergibt das optische Modell ${ }^{2,7}$

$$
\sigma_{c}=\pi\left(\lambda_{c} / 2 \pi\right)^{2} T_{c},
$$

$T_{c}$ heißt Durchdringungsfaktor.

* z. Zt. Oak Ridge National Laboratory, Oak Ridge, Tennessee, USA.

* Die Arbeit wurde mit Unterstützung der Deutschen Forschungsgemeinschaft ausgeführt.

1 W. Hauser u. H. Feshbach, Phys. Rev. 87, 366 [1952].

2 P. A. Moldauer, Phys. Rev. 135, B 642 [1964].

${ }^{3}$ P. A. Moldauer, Phys. Rev. 136, B 947 [1964].

4 P. A. Moldauer, Rev. Mod. Phys. 36, 1074 [1964].
Unter der Annahme, daß das Verhältnis von mittserer Resonanzbreite $\left\langle\Gamma_{\lambda c}\right\rangle$ zu mittlerem Resonanzabstand $D$ sehr viel kleiner als eins ist:

$$
\left\langle\Gamma_{\lambda c}\right\rangle / D \ll 1,
$$

gilt für die Durchdringungsfaktoren ${ }^{7}$

$$
T_{c}=2 \pi\left\langle\Gamma_{\lambda c}\right\rangle / D \text {. }
$$

Damit wird aus (1.1) die von Hauser und FeshBACH vorgeschlagene Formel:

$$
\sigma_{c c^{\prime}}^{\mathrm{HF}}=\pi\left(\frac{\lambda_{c}}{2 \pi}\right)^{2} \frac{T_{c} T_{c^{\prime}}}{\Sigma T_{c^{\prime \prime}}} .
$$

Im Gegensatz zur Bohrschen Annahme ergeben die formalen Reaktionstheorien ${ }^{7}$, daß besonders für Einschußenergien, bei denen noch nicht allzu viele $\mathrm{Ka}$ näle offen sind, Ein- und Ausgangskanal nicht als voneinander unabhängig betrachtet werden können. Moldauer berücksichtigt dies durch die von Lane und LyNN ${ }^{8}$ angegebene Breitenfluktuationskorrektur. Gleichzeitig versucht er auch Beiträge höherer Potenzen in $\left\langle\Gamma_{\lambda c}\right\rangle / D$ zu erfassen. Seine in Herleitung und Anwendung kompliziertere Darstellung von (1.1) lautet:

$\sigma_{c c^{\prime}}^{\mathrm{Mol}}=\pi\left(\frac{\lambda}{2 \pi}\right)^{2}\left\{\frac{\left\langle\Theta_{\lambda c}\right\rangle\left\langle\Theta_{\lambda c^{\prime}}\right\rangle}{\sum_{c^{\prime \prime}}\left\langle\Theta_{\lambda c^{\prime \prime}}\right\rangle} W_{c c^{\prime}}-\frac{\delta_{c c^{\prime \prime}}}{4}\left\langle\Theta_{\lambda c}\right\rangle^{2}\right\}$.

Darin steht $W_{c c^{\prime}}$ für die Breitenfluktuationskorrektur. Durch die Beziehung

$$
T_{c}=\left\langle\Theta_{\lambda c}\right\rangle-\frac{1}{4} Q_{c}\left\langle\Theta_{\lambda c}\right\rangle^{2}
$$

5 F. Schmid, Institut für Kernenergetik der Technischen Hochschule Stuttgart, Diplomarbeit 1966.

6 in: F. BühleR u. F. Sснмidt, Forschungsbericht BMwF-FB K 67-90 [1967].

7 A. M. Lane u. R. G. Thomas, Rev. Mod. Phys. 30, 257 [1958].

8 A. M. Lane u. J. E. Lynn, Proc. Roy. Soc. London A 70, 557 [1957]. 
gelangt man von den Durchdringungsfaktoren zu den neuen Größen $\left\langle\Theta_{\lambda c}\right\rangle$. Für die Abhängigkeit der $T_{c}$ von $\left\langle\Gamma_{\lambda c}\right\rangle$ schlägt Moldauer ${ }^{9}$ folgende Form vor:

$$
T_{c}=1-\exp \left\{-2 \pi\left\langle\Gamma_{\lambda c}\right\rangle / D\right\} .
$$

Die Größe $Q_{c}$ ist nur schwach energieabhängig. Sie liegt zwischen 0 und 2, wobei der Wert 0 für große $\sum_{c^{\prime \prime}} T_{c^{\prime \prime}}$ angenommen wird. Diese Summe wird groß, wenn für ein ankommendes Neutron viele Kanäle offen sind. Dann geht aber $W_{c c^{\prime}} \rightarrow 1$ für $c \neq c^{\prime}$ und deshalb in der sogenannten Kontinuumsnäherung

$$
\sigma_{c c^{\prime}}^{\mathrm{Mol}} \rightarrow \sigma_{c c^{\prime}}^{\mathrm{HF}} .
$$

Kombiniert man (1.7) und (1.6) und summiert über alle möglichen Ausgangskanäle, so ergibt sich, daß $\sigma_{c}$ erhalten bleibt. Die beiden Theorien spalten den Querschnitt für die Bildung des Compoundkerns lediglich verschieden auf. Dabei gilt:

$$
\sigma_{c c}^{\mathrm{Mol}}>\sigma_{c c}^{\mathrm{HF}}
$$

und eine umgekehrte Beziehung für den inelastischen Anteil.

Bei Energien, bei denen noch nicht allzu viele Kanäle offen sind (ein Kanal soll hier erst offen sein, wenn $T_{c}>10^{-3}$ geworden ist) unterscheiden sich (1.5) und (1.6) am stärksten. Man erwartet deshalb auch hier die merkbarsten Abweichungen von (1.9). Bei diesen Energien überwiegt in der Regel der elastische Anteil $\sigma_{c c}$ die inelastischen Anteile. Geht man von der Hauser-Feshbach-Theorie zu der von Moldauer über, so hat das bei einer verhältnismäßig kleinen Vergrößerung von $\sigma_{c c}$ nach (1.10) zur Folge, daß, da ja $\sigma_{c}$ konstant bleibt, die Summe der inelastischen Querschnitte meist relativ stark abnimmt. Ein Vergleich der nach den beiden Theorien berechneten Werte der $\sigma_{c c^{\prime}}$ mit Experimenten wird dann zugunsten der Theorie ausfallen, für die der gesamte inelastische Anteil den Meßwerten am nächsten kommt, er ist also abhängig von den verwendeten Parametern des optischen Modells. Solange keine verbindlichen Kriterien für die Auswahl eines bestimmten Parametersatzes bestehen, kann man vernünftigerweise nur dann Aussagen über die Brauchbarkeit beider Theorien machen, wenn man zwei verschiedene in bezug auf den totalen inelastischen Querschnitt gleichwertige Parametersätze bestimmt und mit den daraus berechenbaren Durch-

9 P. A. Moldauer, Phys. Rev. 157, 907 [1967].

10 F. Sснмidt u. F. Bühler, J. Nucl. Eng. [1967], im Druck. dringungsfaktoren nach (1.5) bzw. (1.6) die zu vergleichenden Wirkungsquerschnitte bildet. Wir haben früher ${ }^{10}$ darauf hingewiesen, daß nach solch modifizierten Anpassungen durch die gemessenen inelastischen Querschnitte keine der Theorien ausdrücklich auszuschließen ist.

Von diesen Voraussetzungen ausgehend wollen wir im vorliegenden Artikel zeigen, wo die beiden Theorien auch bei bestmöglichen Anpassungen unterschiedliche Ergebnise liefern. Wir führen dazu ein einfaches Zweikanalmodell ein, von dem ausgehend wir unsere Überlegungen auf realistischere Fälle erweitern.

Es wird sich zeigen, daß bei den betrachteten niederen Energien das Verhalten von $\sigma_{c}$ und entsprechend die Durchdringungsfaktoren bei kleinen $l$-Werten stark davon abhängen, welche Theorie bei der Bestimmung der Parameter des optischen Modells mitverwendet wurde. Rechnungen an ${ }^{238} \mathrm{U}$ bestätigen dieses Ergebnis und seinen erwarteten numerischen Wert der Größenordnung 2

$$
\sigma_{c}^{\mathrm{Mol}} \sim 2 \sigma_{c}^{\mathrm{HF}} \quad \text { (bei niederen Energien). }
$$

Das gefundene Verhalten von $\sigma_{c}$ ist gut geeignet, einige Diskrepanzen zwischen Messungen und $\mathrm{Be}$ rechnungen inelastischer Querschnitte nach dem Verdampfungsmodell bei niederen Energien der Sekundärneutronen zu beseitigen. Da das verwendete $\mathrm{Zwei}$ kanalmodell auch eine recht einfache Bestätigung der Behauptung, die Moldauersche Theorie gelte bis in Bereiche $\left\langle\Gamma_{\lambda c}\right\rangle / D \leqq 1$, liefert, hoffen wir, mit diesem Artikel die Argumente für diese Theorie zu erhärten, und wir versuchen, über unsere Ergebnisse ein neues Kriterium zur Bestimmung der Parameter des optischen Modells anzugeben.

\section{Kurze Beschreibung des Modells}

Ein durch einen Kanal $c$ einlaufendes Neutron soll nur über zwei Kanäle ( $c$ und $c^{\prime}$ ) den Zwischenkern verlassen können. Ein Beispiel dafür ist die Streuung an Kernen mit Spin $I=0$ bei Energien, durch die im wesentlichen nur das erste Niveau angeregt wird (z.B. bei ${ }^{238} \mathrm{U}$ bis rund $200 \mathrm{keV}$, solange $\sigma_{n^{\prime}}^{2+} \gg \sigma_{n^{\prime}}^{4+}$ und die $T_{l}^{j}$ für $l \geqq 3, j \geqq 2,5$ ungefähr Null sind). Für den Zweikanalfall hat DresNER ${ }^{11}$ als Breitenfluktuationskorrektur folgenden

11 L. Dresner, CU 175 [1957]. 
Ausdruck angegeben:

$W_{c c^{\prime}}=\left\langle\frac{\Theta_{\lambda c} \Theta_{\lambda c^{\prime}}}{\Sigma \Theta_{\lambda c^{\prime \prime}}}\right\rangle / \underset{\left\langle\Theta_{\lambda c}\right\rangle\left\langle\Theta_{\lambda c^{\prime \prime}}\right\rangle}{\left\langle\Theta_{\lambda c^{\prime}}\right\rangle}=(1+z) /\left(1+\sqrt{z)^{2}}{ }^{2}\right.$

wobei $z=\left\langle\Gamma_{\lambda c^{\prime}}\right\rangle /\left\langle\Gamma_{\lambda c}\right\rangle$ und $\left\langle\Gamma_{\lambda c}\right\rangle \ll D$, so daß die Breiten einer Porter-Thomas-Verteilung ${ }^{12}$ genügen :

$$
P\left(\frac{\Gamma_{\lambda c}}{\Gamma_{\lambda}}\right)=\left(2 \pi \frac{\Gamma_{\lambda c}}{\Gamma_{\lambda}}\right)^{-1 / 2} \exp \left\{-\frac{1}{2} \frac{\Gamma_{\lambda c}}{\Gamma_{\lambda}}\right\} .
$$

Alle Wirkungsquerschnitte, die nach diesem Modell berechenbar sind, erhält man aus (1.5) und (1.6), wenn man dort für die Summen schreibt:

$\sum_{c^{\prime \prime}} T_{c^{\prime \prime}}=T_{c}+T_{c^{\prime}}$ und $\sum_{b^{\prime \prime}}\left\langle\Theta_{\lambda c^{\prime \prime}}\right\rangle=\left\langle\Theta_{\lambda c}\right\rangle+\left\langle\Theta_{\lambda c^{\prime}}\right\rangle$.

Wir wollen nun annehmen, es sei uns gelungen, zwei Parametersätze für das optische Potential zu finden, derart, daß jeweils für eine der Theorien der gemessene und berechnete inelastische Anteil innerhalb der Fehlergrenzen übereinstimmen. Die zugehörigen Durchdringungsfaktoren seien die $\Gamma_{c}$ bzw. $\Gamma_{c}^{(1)}$, aus denen nach (1.7) die $\left\langle\Theta_{\lambda c}\right\rangle$ und die $\left\langle\Theta_{\lambda c}^{(1)}\right\rangle$ gebildet werden können.

Um überschaubare mathematische Beziehungen zu erhalten, wollen wir zunächst annehmen, es sei nur ein einziger Eingangskanal $c$ erlaubt. Diese Annahme ist etwas unrealistisch, sie wird später wieder aufgehoben. Nun läßt sich die Anpassungsbedingung des inelastischen Querschnitts einfach anschreiben:

$$
\sigma_{c c^{\prime}}^{\mathrm{HF}}=\sigma_{c c^{\prime}}^{\mathrm{Mol}}=\sigma_{n^{\prime}}^{\mathrm{exp}} .
$$

dem gemessenen Wert. Daraus erhält man durch Einsetzen von (1.5) und (1.6)

$$
W_{c c^{\prime}}^{(1)} \frac{\left\langle\Theta_{\lambda c}^{(1)}\right\rangle\left\langle\Theta_{\lambda c^{\prime}}^{(1)}\right\rangle}{\left\langle\Theta_{\lambda c}^{(1)}\right\rangle\left\langle\Theta_{\lambda c^{\prime}}^{(1)}\right\rangle}=\frac{T_{c} T_{c^{\prime}}}{T_{c}+T_{c^{\prime}}} .
$$

Es soll zunächst der Einfluß der Breitenfluktuationskorrektur, also der Übergang von den $T_{c}$ zu den $T_{c}^{(1)}$ untersucht werden. Die Betrachtungen sollen dann um den Fall mehrerer Eingangskanäle und endlich auch auf den mehrerer Ausgangskanäle erweitert werden. Im 5. Abschnitt werden wir einige Überlegungen zum Übergang von den $T_{c}^{(1)}$ zu den $\left\langle\Theta_{\lambda c}^{(1)}\right\rangle$ anschließen.

12 Jahnke-Emde, Tafeln höherer Funktionen, Teubner, Stuttgart 1966.

\section{Der Einfluß der Breitenfluktuationskorrektur}

Wir betrachten Fälle, für die

$$
\left\langle\Theta_{\lambda c}\right\rangle \approx T_{c} \approx\left\langle\frac{2 \pi \Gamma_{\lambda c}}{D}\right\rangle=\frac{2 \pi\left\langle\Gamma_{\lambda c}\right\rangle}{D}
$$

gilt. Wir setzen an

$$
\begin{aligned}
& T_{c}^{(1)}=\left(T_{c}^{(1)} / T_{c}\right) T_{c}=a T_{c}, \\
& T_{c^{\prime}}^{(1)}=\left(T_{c^{\prime}}^{(1)} / T_{c^{\prime}}\right) T_{c^{\prime}}=b T_{c^{\prime}} .
\end{aligned}
$$

Dann wird aus (2.3) mit (2.1)

$$
\frac{1+z}{(1+\sqrt{z})^{2}} \cdot \frac{b T_{C^{\prime}}}{1+z}=\frac{T_{c^{\prime}}}{1+p},
$$

wo jetzt

$$
z=T_{c^{\prime}}^{(1)} / T_{c}^{(1)}=(b / a) p .
$$

(3.3a) kann man nach $a$ oder $b$ auflösen:

$$
\begin{aligned}
& a=\frac{b p}{(b+b p-1)^{2}}(b+b p+1+2 \sqrt{b+b p)}, \quad, \\
& b=\frac{a}{(a+a p-p)^{2}}\left(a+a p+p+2 \sqrt{\left.\left(p^{2}+p\right) a\right) .}\right.
\end{aligned}
$$

Für die Wurzel ist entsprechend dem positiven Vorzeichen von $\sqrt{z}$ aus (2.1) das signum + zu wählen. Die Kurven (3.3) unterliegen einigen ihren Definitionsbereich beschränkenden, physikalischen Nebenbedingungen.

So gilt, da die Durchdringungsfaktoren $\geqq 0$ und $\leqq 1$ sind,

$$
a, b, p \geqq 0, \quad a T_{c}, b T_{c^{\prime}} \leqq 1 .
$$

Ferner ist aus (2.1) leicht zu ersehen, daß

$$
W_{c c^{\prime}}^{(1)} \leqq 1
$$

und deshalb

$$
a b /\left(a T_{c}+b T_{c^{\prime}}\right) \geqq 1 /\left(T_{c}+T_{c^{\prime}}\right)
$$

oder auch

$$
p(1-1 / a) \geqq(1 / b-1) .
$$

Diese Relation kann in zwei Teilforderungen zerlegt werden.

Für $a \geqq 1$ muß gelten

$$
b \geqq 1 \quad \text { bei beliebigem } p
$$

$1 / T_{c^{\prime}} \geqq b<1 \quad$ bei $\quad p>\frac{a(1-b)}{b(a-1)}$.

Es ist dann $T_{c}^{(1)}>T_{c}$. Wegen $W_{c c}^{(1)}>1$ würde der Compound-elastische Anteil $\sigma_{c c}$ erheblich zunehmen. Um den gleichen Betrag müßte der gesamte Compoundkernquerschnitt anwachsen. 
Für $a<1$ dagegen fordert (3.5b)

$$
1 / T_{c^{\prime}} \geqq b>1 \quad \text { bei } \quad p<\frac{(b-1) a}{(a-1) b} .
$$

Tritt dieser Fall ein, dann wird $\sigma_{c}$ verringert. Entsprechendes gilt für den compoundelastischen Anteil. Aus (2.1) folgt, daß $W_{c c^{\prime}}^{(1)}$ am stärksten von 1 abweicht, wenn $z$ in der Größenordnung 1 liegt $(0,01$ $<z<100)$. Im realistischen Fall, wenn mehrere Zweikanalfälle konkurrieren, sind die $\Gamma_{c}$-Werte, welche die großen Beiträge liefern, größer als 0,01 . Deshalb liegt auch $p$ im für $z$ angegebenen Bereich. Aus diesen Gründen brauchen wir nicht allzu viel Gewicht auf die Betrachtung extremer Werte von $a$ und $b$ zu legen.

In Abb. 1 haben wir $a$ als Funktion von $p$ bei verschiedenen $b$ aufgetragen. Die Kurve mit $b=\infty$ kann wegen $b T_{c^{\prime}} \leqq 1$ schon nicht mehr erreicht werden. $a=1$ erweist sich als Asymptote für große $p$.

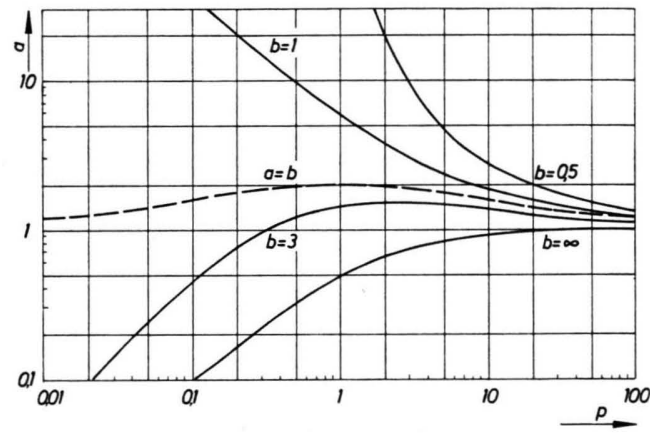

Abb. 1. $a$ als Funktion von $p$ bei verschiedenen Parametern $b$.

Ihr nähern sich alle physikalisch möglichen Kurven von oben. Im von $b<\infty, b \geqq 1$ und $p>0$ eingeschlossenen Gebiet existiert zu jeder Kombination $b, p$ ein eindeutiger Wert $a$. Oberhalb von $b=1$ liegt das durch (3.5c) aufgespaltene Gebiet. Hier existiert zu jedem $b \leqq 1$ eine senkrechte Asymptote. Es ist $p=0$ für $b=1$ und $p=1$ für $b=0,5$. Allgemein gilt:

$$
p_{\text {as }}=\lim _{a \rightarrow \infty} \frac{1-b}{b} \frac{a}{a-1}=\frac{1-b}{b} .
$$

Es können nur Werte angenommen werden, die rechts von dieser Asymptote liegen.

Die Kurve $b=3$ ist typisch für die Kurven des durch (3.5d) charakterisierten Gebietes. Bemerkt sei hierzu, daß der Übergang von $a<1$ nach $a>1$ schon bei relativ niederen $p$-Werten erfolgt.
Wir haben in Abb. 1 auch die Kurve

$$
a=b=\frac{(1+\sqrt{p})^{2}}{1+p}=\left(W_{c c^{\prime}}\right)^{-1}
$$

eingetragen. Es wird sich zeigen, daß bei mehreren Eingangskanälen die vorkommenden Werte von $a$ und $\mathrm{b}$ im wesentlichen aus dem von $a=1$ und $b=1$ begrenzten Gebiet stammen, wobei (3.7) eine ungefähre Schwerpunktslinie bildet. Um dies leichter einzusehen, ist es nützlich, zwei Beispiele für (3.5d) näher zu betrachten. Zunächst sei $T_{c}{ }^{\prime}\left(E^{\prime}\right)>T_{c}(E)$. Dann ist $p>1$ und daraus folgt, daß bei $a<1$ $b>6$ sein muß. Da andererseits auch $b T_{c^{\prime}}<1$ gilt, kann das nur für $T_{c^{\prime}}$-Werte erfüllt werden, die kleiner $1 / 6$ sind. Ist umgekehrt $T_{c}{ }^{\prime}\left(E^{\prime}\right)<T_{c}(E)$, so sind die Beschränkungen nicht ganz so schwer. Eine obere Grenze für die $T_{c^{\prime}}\left(E^{\prime}\right)$ erhält man nun, wenn man für $T_{c}(E)$ den maximalen Wert $T_{c}=1$ einsetzt. Dann kann man wegen $p=T_{c^{\prime}} / T_{c}=T_{c^{\prime}} /\left(T_{c}=1\right)$ für $b T_{c^{\prime}} \leqq 1$ auch schreiben $p b \leqq 1$. Aus Abb. 1 findet man $p \leqq \frac{1}{3}$ und deshalb ist dieser Fall höchstens für Werte $T_{c^{\prime}}<\frac{1}{3}$ realisierbar.

\section{Verallgemeinerungen des Modells}

Um dem Modell realistischere Züge zu geben, wollen wir zunächst mehrere Eingangskanäle zulassen, von denen aus der Compoundkern über je zwei Ausgangskanäle zerfallen kann. Wir können damit schon Reaktionen wie die am Anfang des 3. Abschnitts beschriebene inelastische Streuung von Neutronen an ${ }^{238} \mathrm{U}$ berechnen. Die Anpassungsbedingung (2.3) geht in

$$
\sum_{c} W_{c^{\prime \prime}}^{(1)} g_{c} \frac{a T_{c} b T_{c^{\prime}}}{a T_{c}+b T_{c^{\prime}}}=\sum_{c} g_{c} \frac{T_{c} T_{c^{\prime}}}{T_{c}+T_{c^{\prime}}}
$$

über, wo $g_{c}$ ein mit dem Eingangskanal $c$ verbundener Gewichtsfaktor ist. Zusätzlich zu (4.1) ist zu bedenken, daß Durchdringungsfaktoren zu bestimmten $l$ und $j$, wenn sie nur an verschiedenen Energiestellen genommen oder auf verschiedene Einschuß. energien bezogen werden, als Durchdringungsfaktor sowohl von Eingangskanälen als auch von Ausgangskanälen auftreten können. Hat man z. B. für eine Energiestelle $E=E_{1}$ und das dazugehörige $E^{\prime}=E_{2}$ $a$ und $b$ bestimmt, so muß, wenn man statt mit $E_{1}$ mit $E_{2}$ einschießt, das zu dieser Einschußenergie gehörende $a_{2}$ genau so groß wie das oben berechnete $b_{1}$ sein, wenn nur Spin und Parität beider Kanäle übereinstimmen. Man kann auf diese Weise „Ket- 
ten" konstruieren. In Abb.2 haben wir ein Beispiel dafür gegeben. Ihm entnehmen wir

$$
a_{2}=b_{1} \text {. }
$$

Im allgemeinen wachsen die Durchdringungsfaktoren bei den betrachteten niederen Energien monoton. Um aus den entsprechenden glatten Kurven andere

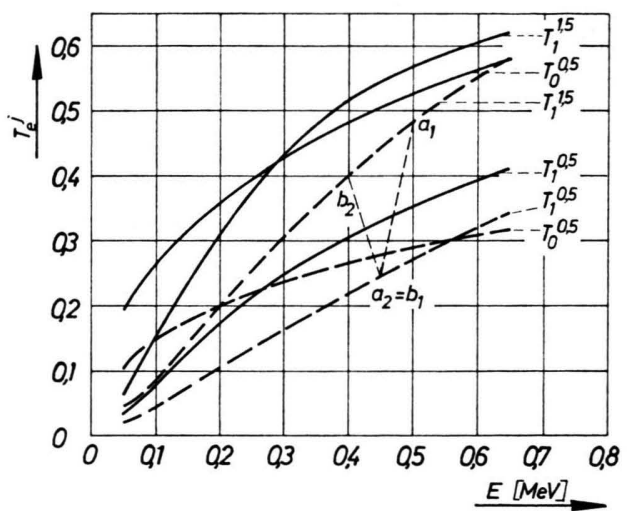

Abb. 2. Durchdringungsfaktoren für ${ }^{238} \mathrm{U}$ bei verschiedenen $l$ und $j$; berechnet über Parameter für Moldauersche Theorie, - - - berechnet über Parameter für HAUSER-F ESHвасн-Theorie. Die Unterschiede betragen einen Faktor 1,5 bis 2. Die Größen $a_{i}, b_{i}$ beziehen sich auf die in Abschn. 5 beschriebenen „Ketten“.

glatte zu erhalten, muß in kleinen Energieabschnitten gelten:

$$
a_{1} \approx b_{2} \text {. }
$$

Gleichzeitig bestehen zwischen den $a_{i}$ und $b_{i}$ Beziehungen der Art (3.3.b). Damit ergibt sich folgendes Schema:

$$
\begin{aligned}
& a_{1} \approx b_{2} \\
& \Downarrow \\
& b_{1}=a_{2} .
\end{aligned}
$$

Die Beziehungen zwischen den $a_{i}$ und $b_{i}$ sind durch die Pfeile angedeutet. Über sie wissen wir aus der Diskussion in Abschn. 3 und aus Abb. 1, daß mit $a_{i}, b_{j} \leqq 1$ immer $b_{i}, a_{j}>1$ verbunden ist, wobei $>1$ meist sogar durch $>2$ ersetzt werden kann. Ferner haben die $b_{i}$ eine obere Grenze in der Gegend von 3 . Versucht man nun, für $a_{i}$ oder $b_{j}$ Werte $<1$ einzusetzen, so gerät man in Widerspruch zu Schema (4.4). Dagegen ergeben die Diskussionen im Abschn. 3, daß (4.4) am ehesten erfüllbar ist, wenn $a_{1} \approx b_{1}$ und $a_{2} \approx b_{2}$ gilt. Solange man also (4.1) als Überlagerung relativ unabhängiger Zweikanalfälle betrachten kann, und wenn $T_{c}$ und $T_{c^{\prime}}$ gleiche Größenordnung haben, folgt aus Abb. 1:

$$
a_{i} \approx b_{i} \leqq 2 \text {. }
$$

Die gemachten Annahmen sind dicht über der Schwelle des ersten Niveaus bei ${ }^{238} \mathrm{U}$ am ehesten erfüllt. Man erwartet daher bei diesem und bei entsprechenden Kernen, daß Durchdringungsfaktoren mit niederen $l$-Werten bei geringen Energien sich um einen Faktor zwei unterscheiden, wenn sie über die verschiedenen Theorien berechnet werden. Dasselbe gilt für den Compoundquerschnitt $\sigma_{c}$ :

$$
\sigma_{c}^{\mathrm{Mol}} \approx 2 \sigma_{c}^{\mathrm{HF}} .
$$

Für größere Energien, bei denen die Zahl der Eingangskanäle zunimmt, wird (4.1) immer mehr abgeschwächt. Dem Übergang $a, b \rightarrow 1$ für hohe Energien entsprechend, sollte (4.6) durch folgende Ungleichung ersetzbar werden:

$$
\sigma_{c}^{\mathrm{HF}} \leqq \sigma_{c}^{\mathrm{Mol}}<2 \sigma_{c}^{\mathrm{HF}} .
$$

Lassen wir neben verschiedenen Eingangskanälen auch mehr als je zwei Ausgangskanäle zu, so wird sich dieses Verhalten noch stärker zeigen. Da jetzt die $W_{c c^{\prime}}^{(1)}$ immer mehr gegen 1 streben, gehen die (3.5) entsprechenden Ungleichungen immer mehr in Gleichungen über: In (4.7) nähert sich $\sigma_{c}^{\mathrm{Mol}}$ an $\sigma_{c}^{\mathrm{HF}}$ an, die beiden Parametersätze werden identisch.

\section{Der Einfluß höherer Glieder in $\Gamma_{\lambda c} / D$}

Höhere Glieder in $\Gamma_{\lambda c} / D$ müssen gemäß (1.8) (dann sind sie physikalischen Ursprungs) oder (1.7) (dann sind sie eine Folge mathematischer Umformungen) berücksichtigt werden. Beide Ursachen wirken einander entgegen. Es ist nicht einfach, die explizite Abhängigkeit $\Theta_{\lambda c}=\Theta_{\lambda c}\left(\Gamma_{\lambda c} / D\right)$ anzugeben ${ }^{3}$. Sie ist wegen ihrer Kompliziertheit auch wenig geeignet zur Berechnung von Wirkungsquerschnitten.

Wir näherten sie deshalb durch eine Potenzreihe an, die so gewählt wurde, daß der Mittelwert $\left\langle\Theta_{\lambda c}\left(\Gamma_{\lambda c} / D\right)\right\rangle$ gerade (1.7) und (1.8) genügt. Wenn die Breiten nach (2.2) verteilt sind, ergibt sich mit

$$
\begin{aligned}
x_{c}=2 & \pi \Gamma_{\lambda c} / D \text { und } \bar{x}_{c}=2 \pi\left\langle\Gamma_{\lambda c} \mid D\right\rangle \approx 2 \pi\left\langle\Gamma_{\lambda c}\right\rangle / D \\
T_{c} & =\bar{x}_{c}-\frac{\bar{x}_{c}^{2}}{2 !}+\frac{\bar{x}_{c}^{3}}{3 !}-+\ldots \\
& =\left\langle x_{c}-\frac{1}{2 ! 3 ! !} x_{c}^{2}+\frac{1}{3 ! 5 ! !} x_{c}^{3}-+\ldots\right\rangle(5.1) \\
& =\left\langle\sum_{n=1}^{\infty}(-1)^{n-1} \frac{x_{c} n}{n !(2 n-1) ! !}\right\rangle \\
T_{c}^{2} & =\bar{x}_{c}^{2}-\bar{x}_{c}^{3}+-\ldots \\
& =\left\langle\frac{1}{3 ! !} x_{c}^{2}-\frac{1}{5 ! !} x_{c}^{3}+\ldots\right\rangle .
\end{aligned}
$$


Etwas komplizierter ist der Übergang zu den $\left\langle\Theta_{\lambda c}\right\rangle$ nach (1.7), da die $Q_{c}$ von $\sum_{c^{\prime \prime}} \Gamma_{\lambda c^{\prime \prime}} / D$ in nicht ganz einfacher Weise abhängen.

Man kann abschätzen ${ }^{3}$

$$
Q_{c}=\frac{2 B_{c}}{N^{2}}\left\{1-\Phi_{0}\left(\sum_{c^{\prime \prime}} \bar{x}_{c^{\prime \prime}}\right)\right\} .
$$

Darin ist $B_{c} / N^{2} \approx 1$ und für $\Phi_{0}(x)$ gilt:

$$
\begin{aligned}
\Phi_{0}(x)=1- & \frac{2}{x}\left[1-\frac{1}{x}\left(1-e^{-x}\right)\right] \\
- & \frac{1}{x}\left[e^{x / 2}+e^{-x / 2}-\frac{2}{x}\left(e^{x / 2}-e^{-x / 2}\right)\right] \\
& \operatorname{Ei}(-x / 2) .
\end{aligned}
$$

Entwickelt man nach Potenzen von $x$ und nähert man die Ei-Funktion $\operatorname{Ei}(-x / 2)$ im Gebiet $0,1 \leq x$ $\leqq 1$ durch $-0,2 / x$, so erhält man für den betrachteten Zweikanalfall

$$
\Phi_{0} \approx \frac{1}{30}+\frac{x_{c}+x_{c^{\prime}}}{3}
$$

und damit

$$
\frac{1}{4} Q \approx \frac{1}{6}\left\{2,9-x_{c}-x_{c^{\prime}}\right\} .
$$

Nun kann man die $\Theta_{\lambda c}$ wie folgt schreiben:

$\Theta_{\lambda c}(x)=x_{c}-\frac{1}{180} x_{c}{ }^{2}-\frac{1,15}{5 ! !} x_{c}{ }^{3}-\frac{1}{18} x_{c}{ }^{2} x_{c^{\prime}}$.

In erster Näherung heben sich die beiden bezüglich $\Theta_{\lambda c}$ gemachten Korrekturen ungefähr auf, ein Ergebnis, auf das wir im 6. Abschnitt zurückkommen werden.

Nun läßt sich bilden:

$$
\begin{aligned}
\frac{\Theta_{\lambda c} \Theta_{\lambda c^{\prime}}}{\Theta_{\lambda c}+\Theta_{\lambda c^{\prime}}} & \approx \frac{x_{c} x_{c^{\prime}}\left(1-\frac{1,15}{5 ! !} x_{c}{ }^{2}-\frac{1}{18} x_{c} x_{c^{\prime}}\right)\left(1-\frac{1,15}{5 ! !} x_{c^{\prime}}{ }^{2}-\frac{1}{18} x_{c} x_{c^{\prime}}\right)}{\left(x_{c}+x_{c^{\prime}}\right)\left(1-\frac{1,15}{5 ! !} \frac{\left(x_{\left.c^{3}+x_{c^{\prime}}\right)}{ }^{3}\right)}{x_{c}+x_{c^{\prime}}}-\frac{1}{18} x_{c} x_{c^{\prime}}\right)} \\
& \approx \frac{x_{c} x_{c^{\prime}}}{x_{c}+x_{c^{\prime}}}\left\{1-\frac{1,15}{5 ! !}\left(x_{c}{ }^{2}+x_{c^{\prime}}{ }^{2}\right)-\frac{1}{18} x_{c} x_{c^{\prime}}+\frac{1,15}{15} \frac{x_{c}{ }^{3}+x_{c^{\prime}}{ }^{3}}{x_{c}+x_{c \prime}}\right\} \\
& =\frac{x_{c} x_{c^{\prime}}}{x_{c}+x_{c^{\prime}}}\left\{1-\frac{1,15}{15}\left(x_{c}{ }^{2}+x_{c^{\prime}}{ }^{2}\right)-\frac{5,14}{18} x_{c} x_{c^{\prime}}+\frac{1,15}{15}\left(x_{c}+x_{c^{\prime}}\right)^{2}\right\} \\
& =\frac{x_{c} x_{c^{\prime}}}{x_{c}+x_{c^{\prime}}}\left\{1+\left(\frac{2,3}{15}-\frac{5,14}{18}\right) x_{c} x_{c^{\prime}}\right\}=\frac{x_{c} x_{c^{\prime}}}{\left(x_{c}+x_{c^{\prime}}\right)}\left\{1-\frac{11,9}{90} x_{c} x_{c^{\prime}}\right\} .
\end{aligned}
$$

Damit kann man Breitenfluktuationskorrekturen für den Zweikanalfall berechnen:

$$
W_{c c^{\prime}} \approx \frac{\left\langle\Theta_{\lambda c}\right\rangle+\left\langle\Theta_{\lambda c^{\prime}}\right\rangle}{\left\langle\Theta_{\lambda c}\right\rangle\left\langle\Theta_{\lambda c^{\prime}}\right\rangle} \int \frac{x_{c} x_{c^{\prime}}}{\left(x_{c}+x_{c^{\prime}}\right)}\left\{1-\frac{11,9}{90} x_{c} x_{c^{\prime}}\right\} P\left(\frac{x_{c}}{\bar{x}_{c}}\right) P\left(\frac{x_{c^{\prime}}}{\bar{x}_{c^{\prime}}}\right) \mathrm{d}\left(\frac{x_{c}}{\bar{x}_{c}}\right) \mathrm{d}\left(\frac{x_{c^{\prime}}}{\bar{x}_{c^{\prime}}}\right) .
$$

$P(x)$ wurde mit (2.2) eingeführt.

Mit den von DresNer ${ }^{11}$ angegebenen Methoden erhält man für die Breitenfluktuationskorrektur

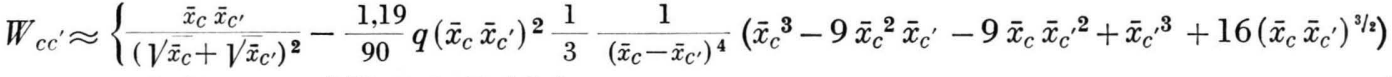

$$
\begin{aligned}
& =\frac{\bar{x}_{c}+\bar{x}_{c^{\prime}}}{\left(\sqrt{\bar{x}_{c}}+\sqrt{\left.\bar{x}_{c^{\prime}}\right)^{2}}\right.}-\frac{1,19}{3} \frac{\bar{x}_{c} \bar{x}_{c^{\prime}}\left(\bar{x}_{c}+\bar{x}_{c^{\prime}}\right)}{\left(\bar{x}_{c}-\bar{x}_{c^{\prime}}\right)^{4}}\left\{\bar{x}_{c^{\prime}}{ }^{3}+\bar{x}_{c}^{3}-9 \bar{x}_{c} \bar{x}_{c^{\prime}}\left(\bar{x}_{c}+\bar{x}_{c^{\prime}}\right)+16\left(\bar{x}_{c} \bar{x}_{c^{\prime}}\right)^{3 / 2}\right\} \text {. }
\end{aligned}
$$

Im Grenzfall $\bar{x}_{c} \rightarrow \bar{x}_{c^{\prime}}$ strebt der erste Bruch gegen sein Minimum und der zweite Ausdruck gegen $0,3 \bar{x}_{c}^{2}$. Solange die $T_{c}$ durch Reihen bis $\bar{x}_{c}^{2}$ genügend gut genähert werden, ist das eine kleine Korrektur und die $W_{c c^{\prime}}$ wurden, wie aus der Entwicklung der $\Theta_{\hat{\lambda} \text { c }}$ zu erwarten war, kaum verändert. Dementsprechend können die Überlegungen der vorherigen Abschnitte weiterhin übernommen werden.

\section{Anwendungen}

Wir haben für das Beispiel ${ }^{238} \mathrm{U}$ nach zwei die Bedingungen von Abschn. 1 erfüllenden Parametersätzen des optischen Modells gesucht. Es stand uns folgende Potentialform ${ }^{13}$ programmiert zur Verfügung:

$$
\begin{aligned}
V= & -V_{0}(E) f(r)-i W_{0}(E) e^{-[(r-R) / b]^{2}} \\
& +\frac{1}{r} \frac{\mathrm{d}}{\mathrm{d} r}\left\{\left(V S_{0}+i W S_{0}\right) f(r)\right\} \sigma l, \\
f(r)= & \left\{1+e^{(r-R) / a}\right\}^{-1} \\
R= & R_{0} A^{1 / 3}
\end{aligned}
$$

Eine Anpassung gelang uns für Energien zwischen $50 \mathrm{keV}$ und $1,5 \mathrm{MeV}$.

13 F. BühLER, Helv. Phys. Acta 37, 241 [1964]. 
Als Parameter ${ }^{6}$ erhielten wir bei Verwendung der Hauser-Feshbach-Theorie

$$
\begin{gathered}
V_{0}=41,5 \mathrm{MeV} ; \quad W_{0}=6,79+1,7 E \mathrm{MeV} ; \\
V S_{0}=15 \mathrm{MeV} ; \quad W S_{0}=0 ; \\
a=0,47 ; \quad b=1 ; \quad R_{0}=1,32 \mathrm{f}
\end{gathered}
$$

und bei der nach Moldauer

$$
\begin{gathered}
V_{0}=42,278-2,778 E \mathrm{MeV} ; \\
W_{0}=12,667-3,333 E \mathrm{MeV} ; \\
V S_{0}=15 \mathrm{MeV} ; \quad W S_{0}=0 ; \\
a=0,66 ; \quad b=1 ; \quad R_{0}=1,25 \mathrm{f} .
\end{gathered}
$$

Es ist uns nicht gelungen, Parametersätze zu finden, die für höhere Einschußenergien ineinander übergehen. Trotzdem zeigen die in Abb. 3 aufgezeichneten Compoundkernbildungsquerschnitte $\sigma_{c}^{\mathrm{Mol}}$ und $\sigma_{c}^{\mathrm{HF}}$

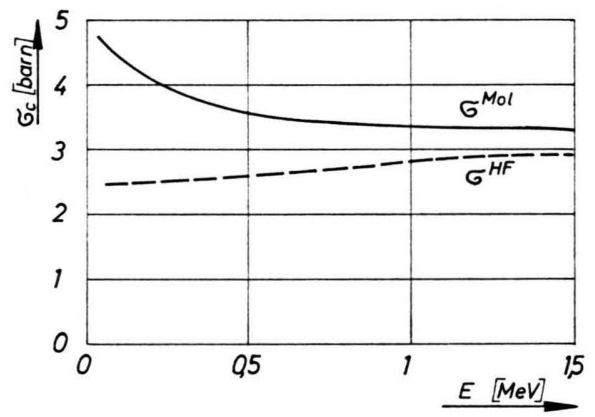

Abb. 3. $\sigma_{c}$ bei ${ }^{238} \mathrm{U}$; $\longrightarrow$ nach Moldauer, ---- nach Hauser-Feshbach bei den in Abschn. 6 angegebenen Parametersätzen.

das erwartete Verhalten. Insbesondere nähert sich ihr Verhältnis für kleine Energien, also dann, wenn die in 4. diskutierte Überlagerung von Zweikanalfällen überwiegt, dem Wert 2. Entsprechendes gilt für die Durchdringungsfaktoren in Abb. 2.

Das Anwachsen von $\sigma_{c}$ mit abnehmender Energie wird damit bestätigt. Ein solches Anwachsen ermöglicht es uns, auf eine Verbesserung der Übereinstimmung von gemessenen und nach dem Verdampfungsmodell berechneten Werten der inelastischen Streuung hinzuweisen.

Dort ergibt die Rechnung für das Spektrum der emittierten Neutronen ${ }^{14}$

$$
N(E) \sim E \cdot \sigma_{c}(E) \cdot U^{-2} \exp \left\{2(a U)^{1 / 2}\right\},
$$

wo $E$ die Neutronenenergie und $U$ die Anregungsenergie des Restkerns bedeutet. Trägt man nun gemäß Buccino et al. ${ }^{14}$ (dortige Fig. 3) für feste Einschußenergie $E_{0}$ die gemessene Größe

$$
\ln \left[N(E) U^{2} / E \sigma_{c}(E)\right]
$$

über $U^{1 / 2}$ auf, so erhält man eine Abweichung von der erwarteten Geraden für $U \rightarrow E_{0}$, also für kleine $E$. Die aus den Messungen errechneten Punkte liegen über den erwarteten Werten. Berücksichtigt man nun, daß mit abnehmendem $E \sigma_{c}$ anwächst, so läßt sich diese Abweichung korrigieren.

Zum Schluß möchten wir noch auf die in (5.7) gefundene Abhängigkeit $\Theta_{\lambda c}=\Theta_{\lambda c}\left(\Gamma_{\lambda c}\right)$ eingehen. Solange man nur Glieder zweiter Ordnung zu berücksichtigen braucht, ist

$$
\left\langle\Theta_{\lambda c}\right\rangle \sim\left\langle\Gamma_{\lambda c}\right\rangle
$$

in guter Näherung erfüllt. Bildet man damit das Verhältnis $\left\langle\Theta_{\lambda c^{\prime}}\right\rangle /\left\langle\Theta_{\lambda}\right\rangle$, so erhält man

$$
\left\langle\Theta_{\lambda c^{\prime}}\right\rangle /\left\langle\Theta_{\lambda}\right\rangle=\left\langle\Gamma_{\lambda c^{\prime}}\right\rangle /\left\langle\Gamma_{\lambda}\right\rangle .
$$

Das ist aber genau die in (1.1) erwähnte Wahrscheinlichkeit für den Zerfall des Compoundkerns über den Kanal $c^{\prime}$. Abgesehen von der Breitenfluktuationskorrektur und der damit verbundenen Kopplung von Ein- und Ausgangskanal bleibt der physikalische Gehalt der Bohrschen Vorstellung beim Übergang zur Moldauerschen Theorie erhalten. Die Behauptung, diese Theorie sei auch für Werte $2 \pi \Gamma_{\lambda c} / D \leqq 1$ anwendbar (also wenn quadratische Glieder mitberücksichtigt werden müssen), erhält damit eine anschauliche Bestätigung.

Wir hoffen, mit diesem Vergleich der Theorien von Hauser-Feshbach und P. A. Moldauer gezeigt zu haben, daß trotz der unsicheren Parameter des optischen Modells Gründe dafür bestehen, der MoLDaUerschen Theorie den Vorzug zu geben. Insbesondere scheinen uns Parametersätze, deren zugehörige Durchdringungsfaktoren mit der Moldauerschen Theorie befriedigende Ergebnisse liefern, vor anderen größere Gültigkeit beanspruchen zu können.

14 S. G. Buccino, C. E. Hollandsworth, H. W. Lewis, and P. R. Bevington, Nucl. Phys. 60, 17 [1964]. 